\title{
Flexible Consistency for Wide area Peer Replication
}

\author{
Sai Susarla and John Carter \\ School of Computing, University of Utah \\ $\{$ sai, retrac\}@cs.utah.edu
}

\begin{abstract}
The lack of a flexible consistency management solution hinders $P 2 P$ implementation of applications involving updates, such as read-write file sharing, directory services, online auctions and wide area collaboration. Managing mutable shared data in a P2P setting requires a consistency solution that can operate efficiently over variable-quality failure-prone networks, support pervasive replication for scaling, and give peers autonomy to tune consistency to their sharing needs and resource constraints. Existing solutions lack one or more of these features.

In this paper, we describe a new consistency model for P2P sharing of mutable data called composable consistency, and outline its implementation in a wide area middleware file service called Swarm ${ }^{1}$. Composable consistency lets applications compose consistency semantics appropriate for their sharing needs by combining a small set of primitive options. Swarm implements these options efficiently to support scalable, pervasive, failure-resilient, wide-area replication behind a simple yet flexible interface. We present two applications to demonstrate the expressive power and effectiveness of composable consistency: a wide area file system that outperforms Coda in providing close-to-open consistency over WANs, and a replicated BerkeleyDB database that reaps order-of-magnitude performance gains by relaxing consistency for queries and updates.
\end{abstract}

\section{Introduction}

Organizing wide area applications in a peer-topeer $(\mathrm{P} 2 \mathrm{P})$ fashion can improve availability, failure resilience, and scalability compared to traditional clientserver architectures. Many effective P2P techniques have been developed to locate and share read-only content [13] or low-write-sharing content [3, 10, 14]. Although P2P organization could benefit wide area applications with mutable data such as online auctions, directory services, and collaborative applications (e.g., Lotus Notes), their data characteristics and consistency needs are diverse and inadequately supported by existing P2P middleware systems.

$\mathrm{P} 2 \mathrm{P}$ sharing of mutable data raises the issues of replication and consistency management. P2P systems pose unique challenges to replication algorithms. P2P clients tend to experience diverse network characteristics and vary in their resource availability and willingness to handle load from other peers. The collection of clients tends to be large, but constantly changing. A P2P consistency management system must therefore be able to support pervasive replication to scale with load, enable peers to individually balance consistency and availability against performance and resource usage, and operate efficiently across non-uniform failure-prone networks.

To understand the diversity of application characteristics and their consistency needs, we surveyed a variety of wide-area data sharing applications ranging from personal file access (with little data sharing) to widespread real-time collaboration (e.g., chat and games, with finegrain synchronization) [16]. The survey identified three broad classes of distributed applications: (1) file access, (2) database and directory services, and (3) real-time collaborative groupware.

We found that applications differ widely in the frequency and extent of read and write sharing among replicas, the typical replication factor, their tolerance to stale data, semantic interdependencies among updates, the likelihood of conflicts among concurrent updates, and their amenability to automatic conflict resolution. Some applications need different consistency semantics for reads and writes, e.g., an online auction service might need strong consistency for writes to prevent selling the same item twice, but could tolerate providing stale data in response to queries to improve performance. Applications differ in the degree to which they tolerate replica divergence, e.g., users working on a shared document expect it to reflect the latest updates made by other users before each editing session (close-to-open consistency), while the staleness of stock-quote updates should be bounded by a time interval. Applications access files in different ways with different consistency requirements, e.g., personal files are rarely shared while software and multimedia file are widely read-shared. Other applications frequently write-share data, e.g., distributed logs, shared calendars, and version control systems. How concurrent updates can be handled varies widely, e.g., it is relatively easy to combine concurrent appends (logging) or merge-able writes (calendars), but more complex sharing and write conflict patterns often require explicit serialization. For some applications, optimistic or eventual consistency (i.e., propagating updates lazily) provides adequate semantics and high availability, but during periods of close collaboration users need tighter synchronization guarantees such as close-

${ }^{1}$ Swarm stands for Scalable Wide Area Replication Middleware. 
to-open (to view latest updates) or strong consistency (to prevent update conflicts).

Based on the results of our application study, we believe that managing mutable shared data in a P2P setting requires a consistency solution that can operate efficiently over variable-quality failure-prone networks, support pervasive replication for scaling, and give peers autonomy to tune consistency to their sharing needs and resource constraints. Existing solutions fall short of meeting one or more of these requirements. Several $\mathrm{P} 2 \mathrm{P}$ systems support file and database replication with updates by providing close-to-open or eventual consistency $[10,3,14,12]$, which is inadequate for many applications. A number of previous efforts $[18,6]$ have viewed consistency semantics as a continuous linear spectrum ranging from strong consistency to best-effort eventual consistency, and provided ways to fine-tune divergence of replica contents. While powerful, these systems need to be extended in several ways to cover the full spectrum of observed application needs.

When we carefully examined the results of our survey, we observed that the consistency needs of applications can be expressed in terms of a small number of design options, which we classify into five mostly orthogonal dimensions:

- concurrency - the degree to which conflicting (read/write) accesses can be tolerated,

- replica synchronization - the degree to which replica divergence can be tolerated (called coherence), and the types of inter-dependencies among updates that must be preserved when synchronizing replicas (called consistency),

- failure handling - how data access should be handled when some replicas are unreachable or have poor connectivity,

- update visibility - the time at which local modifications to replicated data become 'stable' and ready to be made visible globally, and

- view isolation - the time at which remote updates must be made visible locally.

There are multiple reasonable options along each of these dimensions that existing systems employ separately. Based on this classification, we developed a novel composable consistency model that provides the options listed in Table 1. When these options are combined in different ways, they yield a rich collection of consistency semantics for shared data that cover the needs of a broad variety of applications. Using composable consistency, a P2P auction service could employ strong consistency for updates, while relaxing consistency for queries to limit synchronization cost. An auction user can specify stronger consistency requirements to ensure $100 \%$ accurate query results, although doing so increases overhead and latency.

In this paper, we describe the composable consistency model and outline how we implemented it in a wide area P2P middleware file service called Swarm. Swarm lets applications express consistency requirements as a vector of options along these dimensions on a per-access basis rather than only supporting a few packaged combinations. Doing so gives applications more flexibility in balancing consistency, availability, and performance. In addition, Swarm performs aggressive peer replication and tunes its replication behavior in response to observed network and node availability conditions. As a result, Swarm more effectively caches data near where it is accessed, thereby providing low latency data access, under a broader range of data sharing scenarios than existing systems.

To determine the value of composable consistency in Swarm, we implemented both a wide area P2P file system and a wrapper library that supports replication around the BerkeleyDB database library [15]. We show that Swarm's composable consistency ensures close-to-open semantics over a WAN and outperforms Coda's client-server implementation by exploiting 'nearby' replicas during roaming file access. We also show that replicating BerkeleyDB using Swarm enables order-of-magnitude improvements in write performance and scalability beyond master-slave replication by relaxing consistency in several ways. Swarm relieves application writers of the burden of implementing their own replication and consistency mechanisms. Since the focus of this paper is on evaluating composable consistency in a wide area $\mathrm{P} 2 \mathrm{P}$ replication environment, we limit our discussion of Swarm's design to its consistency implementation. A more complete description and evaluation of Swarm's design, including its dynamic hierarchical replication and its scalability and failure resilience properties, appears elsewhere [16].

In Section 2 we describe prior work on flexible consistency management and wide area replication management. In Section 3 we describe the composable consistency model. We briefly outline Swarm's design in Section 4 before describing its consistency implementation in Section 5. We present our evaluation of its practicality in Section 6. Finally, in Section 7 we conclude.

\section{Related Work}

Several solutions exist to manage replication among wide-area peers. Several P2P systems were developed to share read-only data such as multimedia files (PAST [13], KaZaa) or access to rarely write-shared personal files [14], but not frequent write-sharing. $\mathrm{Nu}$ merous consistency schemes have been developed individually to handle the data coherence needs of specific services such as file systems, directory services [9], databases and persistent object systems [8]. Distributed 


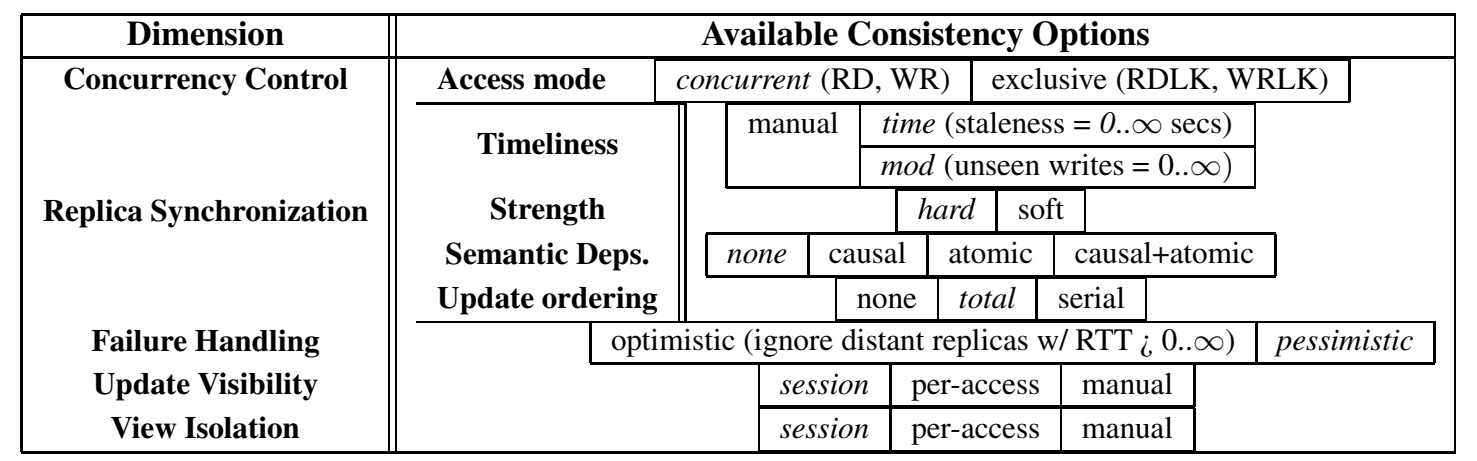

Table 1: Consistency options provided by the Composable Consistency (CC) Model. Consistency semantics are expressed for an access session by choosing one of the alternative options in each row, which are mutually exclusive. Options in italics indicate reasonable defaults that suit several applications. In our discussion, when we leave an option unspecified, we assume its default value.

file systems such as NFS, Pangaea, Sprite, AFS, Coda and Ficus target traditional file access with low writesharing among multiple users. Ivy [10] is a read/write $\mathrm{P} 2 \mathrm{P}$ file system that supports close-to-open semantics based on P2P block storage and logs. Composable consistency adopts a novel approach to support many of their consistency semantics efficiently in a P2P setting.

Bayou [3] explored optimistic replication and update propagation for collaborative applications under ad-hoc connectivity. Our consistency implementation employs some of their techniques in a wider context.

Fluid replication [2] provides multiple selectable consistency policies for static hierarchical caching. In contrast, our approach offers primitive options that can be combined to yield a variety of policies, offering more customizability for peer-to-peer replication. Many design options [7, 6] that we identified for composable consistency are proposed separately by prior systems. Our model forges them uniquely for flexibility.

Many previous efforts have explored a continuous consistency model $[18,6]$. Of those, the TACT toolkit [18] provides continuously tunable consistency along three dimensions similar to those covered by our timeliness and concurrency control aspects. We provide additional flexibility including the notion of sessions and explicit semantic dependencies to cater to a wider variety of application needs as described in Section 3. TACT's order error offers continuous control over the number of update conflicts. In contrast, our concurrency options provide a binary choice between zero and unlimited number of conflicts. We believe that for many real-world applications, a binary choice such as ours is adequate and reduces bookkeeping overhead.

\section{Composable Consistency Model}

The composable consistency (CC) model is applicable to systems that employ the data-shipping paradigm. Such applications are structured as multiple distributed components, each of which holds a portion of appli- cation state and operates on other portions by locally caching them as needed. The $\mathrm{CC}$ model assumes that applications access (i.e., read or write) their data in sessions, and that consistency can be enforced at session boundaries or before and after each read or write access.

In the CC model, an application expresses its consistency requirements for each session as a vector of consistency options, as listed in Table 1. Each row of the table indicates several mutually exclusive options available to control the aspect of consistency indicated in its first column. Reasonable default options are noted in italics, which together enforce the close-to-open consistency semantics provided by AFS [4] for coherent readwrite file sharing. A particular CC implementation can provide reasonable defaults, but allow an application to override the defaults if needed. Table 2 lists the CC options that achieve several consistency flavors.

Concurrency: $\mathrm{CC}$ provides two flavors of access modes to control the parallelism among reads and writes. Concurrent modes (RD, WR) allow arbitrary interleaving of accesses across replicas, similar to Unix file semantics. Exclusive modes (RDLK, WRLK) provide traditional concurrent-read-exclusive-write semantics [11]. When both flavors are employed on the same data simultaneously, RD mode sessions coexist with all other sessions, i.e., RD operations can happen in parallel with exclusive sessions, while WR mode sessions are serialized with respect to exclusive sessions, i.e., they occur before a RDLK/WRLK session begins or are deferred until it ends.

Timeliness Guarantees: The degree to which replicas are allowed to diverge in contents (i.e., timeliness in Table 1) can be specified in terms of time, the number of missed updates, or both. These options are analogous to the TACT toolkit's staleness and numeric error metrics [18]. The timeliness bounds can be hard, i.e., strictly enforced by stalling writes if necessary (like TACT), or soft, i.e., enforced in a best-effort manner for high availability. Two types of semantic dependencies can be di- 
rectly expressed among multiple writes (to the same or different data items), namely, causality and atomicity. These options together with the session abstraction can be used to support the transactional model of computing.

Update Ordering Constraints: When updates are issued independently at multiple replicas (e.g., by 'WR' mode sessions), our model allows them to be applied (1) with no particular constraint on their ordering at various replicas (called 'none'), (2) in some arbitrary but common order everywhere (called 'total'), or (3) sequentially via serialization (called 'serial'). The ordering options can be specified on a per-session basis. The unordered updates option is suitable when updates are commutative, e.g., increments to a shared counter or updates to different entries in a shared directory. Totally ordered updates can be made concurrently, but may need to be reapplied to ensure global ordering. Some updates that require total order cannot be undone and reapplied, and thus must be globally serialized. For example, when multiple clients concurrently issue the dequeue operation at different replicas of a shared queue, they must not obtain the same item, although multiple items can be enqueued concurrently and reordered later. Hence the dequeue operation requires 'serial' ordering, while enqueue requires 'total' ordering.

Failure Handling: When all replicas are not equally well-connected, different consistency options can be imposed on different subsets of replicas based on their relative connectivity by specifying a cut-off network quality. Consistency semantics are guaranteed at a replica only relative to those replicas reachable via higher quality links. For instance, this enables a directory service to provide strong consistency among clients within a campus, while enforcing optimistic/eventual consistency across campuses for higher availability.

Visibility and Isolation: Finally, our model allows control over how long a session stays isolated from the updates of remote sessions, as well as when its own updates are made ready to be visible to remote sessions. A session can remain completely isolated from remote updates ('session', ensuring a snapshot view of data), allow updates to be applied on local copies immediately ('per-access', useful for log monitoring), or explicitly import remote updates ('manual'). Similarly, a session's updates can be propagated as soon as they are issued (useful for chat), when the session ends (useful for file updates), or when explicitly requested.

Although CC's options are largely orthogonal, they are not completely independent. For example, the exclusive access modes imply session-grain visibility and isolation, a hard most-current timeliness guarantee, and serial update ordering.

At first glance, providing a large number of options rather than a small set of hardwired protocols might appear to impose an extra burden on application programmers. However, thanks to the orthogonality and com- posability of CC's options, their semantics are roughly additive; each option only restricts the applicability of the semantics of other options and does not alter them in unpredictable ways. Thus, by adopting our model, programmers are not faced with a combinatorial increase in the complexity of semantics to understand. To further ease the adoption of the CC model for application design, we anticipate that middleware systems adopting it will bundle popular combinations of options as defaults (e.g., 'Unix file semantics', 'CODA semantics', or 'best effort streaming'), while allowing individual applications to refine their consistency semantics when required. In those circumstances, programmers can customize individual options along some dimensions while retaining the other default options. Finally, the CC model allows multiple clients to request different consistency semantics on the same data simultaneously, on a per-access-session basis. In that case, the sharing semantics obtained by each client depends only on the options chosen by that client, regardless of the potentially conflicting choices made by other clients elsewhere. In Section 5, we present our implementation of the model that ensures this property by serializing sessions with conflicting semantics.

\section{Swarm}

In this section, we outline the Swarm distributed file store [16] in which we implemented and evaluated composable consistency. Swarm is organized as a collection of peer servers (called Swarm servers) that provide coherent wide area file access at a variable granularity. Applications store their shared state in Swarm files and operate on their state via nearby Swarm servers.

Files in Swarm are persistent variable-length flat byte arrays named by globally unique 128-bit numbers called SWIDs. Swarm exports a file system-like interface that supports traditional read/write operations on file blocks as well as operational updates on files (explained below). A file block (also called a page, default $4 \mathrm{~KB}$ ) is the smallest unit of data sharing and consistency in Swarm. Swarm servers locate files by their SWIDs, cache them as a side-effect of local access, and maintain consistency according to the per-file consistency options described in Section 3. Each Swarm server uses a portion of its local persistent store for permanent ('home') copies of some files and uses the remaining space to cache remotely homed files. Swarm servers discover each other as a side-effect of locating files by their SWIDs. Each Swarm server monitors the connection quality (latency, bandwidth, connectivity) to other Swarm servers with which it has communicated in the recent past, and uses this information to form an efficient dynamic hierarchical overlay network of replicas of each file.

Interface: Swarm exports the traditional sessionoriented file interface to its client applications via a Swarm client library linked into each application pro- 


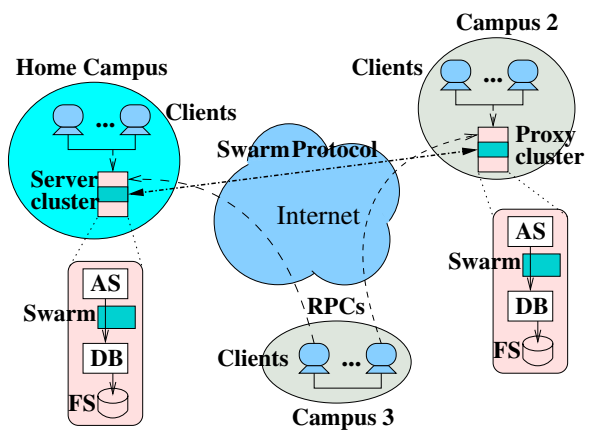

Figure 1: An enterprise application employing a Swarm-based proxy server. Clients in campus 2 access the local proxy server, while those in campus 3 invoke either server.

cess. The interface allows applications to create and destroy files, open a file session with specified consistency options, read and write file blocks, and close a session. A session is Swarm's unit of concurrency control and isolation. A Swarm server also exports a native file system interface to $S$ warm files via the local operating system's VFS layer, similar to CodaFS [5]. Swarm provides a hierarchical file name space by implementing directories within Swarm files.

Swarm allows files to be updated in two ways: (i) by directly overwriting the previous contents on a per file block basis (called absolute or physical updates) or (ii) by registering a semantic update procedure (e.g., "add(name) to directory") and then invoking an update () interface to get Swarm to perform the operation on each replica (called operational updates). Before issuing an operational update, the application first must link a plugin to each Swarm server running an application component. The plugin is used both to interpret and apply update procedures and to perform application-specific conflict resolution. When operational updates are used, Swarm replicates an entire file as a single consistency unit.

Using Swarm: To use Swarm, applications link to a Swarm client library that invokes operations on a nearby Swarm server in response to client operations (e.g., creating a file, opening an access session, or performing a read or write). When applications open a file, they can specify a set of consistency options. As described below, the local Swarm server interacts with other Swarm servers to acquire and maintain a locally cached copy of the file with the specified consistency. Figure 1 illustrates how an auction service and a directory service (both derived from the BerkeleyDB embedded database library [15]) could use Swarm to implement wide area caching proxies. The 'DB' represents the unmodified BerkeleyDB database service oblivious to replication, the 'AS' represents the auction or directory servicespecific logic, and the 'FS' provides local storage. Our evaluation in Section 6 shows the benefit of such replication.

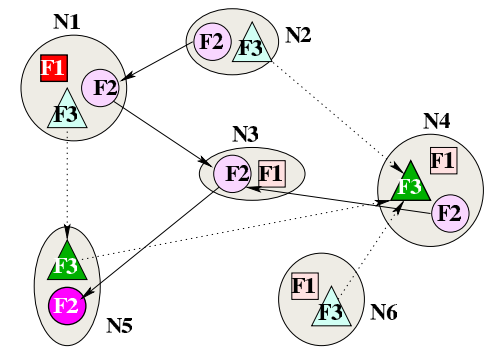

Figure 2: File replication in a Swarm network. Files F1..F3 are replicated at Swarm servers N1..N6. Permanent copies are shown in darker shade. F1 and F2 are homed at N1. F3 has two custodians: N4 and N5. Replica hierarchies are shown for F2 and F3 rooted at N5 and N4 respectively. Arrows indicate parent links.

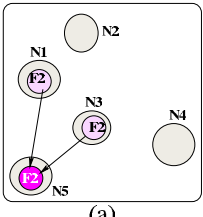

(a)

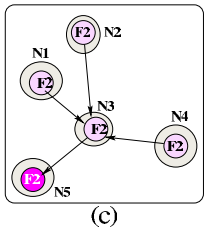

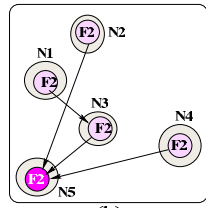

(b)

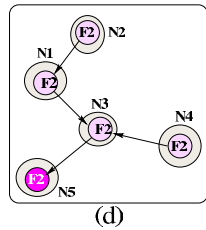

Figure 3: Replication of file F2. (a) N1 and N3 cache F2 from its home N5. (b) N2 and N4 cache it from N5; N1 reconnects to closer replica N3. (c) Both $\mathrm{N} 2$ and N4 reconnect to N3 as it is closer than N5. (d) Finally, N2 reconnects to N1 as it is closer than $\mathrm{N} 3$.

Replication: The Swarm servers caching a particular file dynamically organize themselves into an overlay replica hierarchy. All communication between replicas happens via this hierarchy. Figure 2 shows a Swarm network of six servers with hierarchies for two files. One or more servers act as custodians, which maintain permanent copies of the file. Having multiple custodians enables fault-tolerant file lookup. One custodian, typically the server that created the file, is designated the root custodian or home node, and coordinates the file's replication and consistency management, as described below. When the root fails, another custodian is elected as root by a majority vote among custodians.

The focus of this paper is Swarm's consistency maintenance, so we briefly describe how files are replicated in Swarm, and refer the reader elsewhere [16] for further details on how Swarm creates cycle-free replica hierarchies in the face of node churn, optimizes the replica hierarchy based on observed network characteristics, and provides fault resilience.

Swarm caches files based on local access by clients. Figure 3 illustrates how the hierarchy is formed for file F2 as nodes successively cache it locally. Node dis- 
tances in the figure are roughly indicative of their network distances (roundtrip times). When a Swarm server $\mathrm{R}$ wants to cache a file locally (to serve a local access), R first uses the file's SWID to locate its custodians, e.g., via an external location service such as Pastry [13] or via a simple mechanism like hardwiring the IP address into the SWID, an approach we employ in our prototype. R keeps track of these custodians, and then requests one of them (say P) to be its parent replica and provide a file copy, preferring those that can be reached via high quality (low-latency) links. Unless P is serving "too many" child replicas, $\mathrm{P}$ accepts $\mathrm{R}$ as its child, transfers the file contents, and initiates consistency maintenance (as explained in Section 5). P also sends the identities of its child replicas to $\mathrm{R}$ and indicates if it has reached its configured fanout limit. R augments its lookup cache with the supplied information. If $\mathrm{P}$ was overloaded, $\mathrm{R}$ remembers to avoid asking $\mathrm{P}$ for that file in the near future, otherwise $\mathrm{R}$ sets $\mathrm{P}$ as its parent replica. $\mathrm{R}$ repeats this parent election process until it has a valid file copy and a parent replica (the root custodian is its own parent). Even when a replica has a valid parent, it monitors its network quality to known replicas and reconnects to a closer replica, if found. This process forms a dynamic hierarchical replica network rooted at the root custodian like Blaze's file caching scheme [1], but avoids hops over slow network links when possible, like Pangaea [14]. When a replica loses contact with its parent, it re-elects its parent in a similar manner to reconnect to the hierarchy. To guard against the root's temporary failure, the root propagates the identities of the its direct children to all replicas in the background, so reachable replicas can re-group into a tree until the root recovers.

\section{Implementing Composable Consistency}

In this section, we describe how composable consistency is implemented in Swarm to illustrate its practicality in a wide area replication environment. Each Swarm server has a consistency module (CM) that is invoked when clients open or close a file or when clients perform reads or updates within a session. The CM performs checks, interacting with replica neighbors (parent and children) via pull operations as described below, to ensure that the local file copy meets client requirements. Similarly, when a client issues updates, the CM propagates them via push operations to enforce the consistency guarantees given by peer servers to their clients.

To succinctly track concurrency control and replica divergence guarantees (i.e., time and mod bounds) given to client sessions, the CM internally represents them by a construct called the privilege vector $(P V)$. The $\mathrm{CM}$ can allow a client to access a local replica without contacting peers if the replica's consistency indicated by its PV is at least as "strong" as that required by the client. For example, if a client requires $200 \mathrm{~ms}$ staleness and the PV guarantees a max staleness of $100 \mathrm{~ms}$, no pull is required.

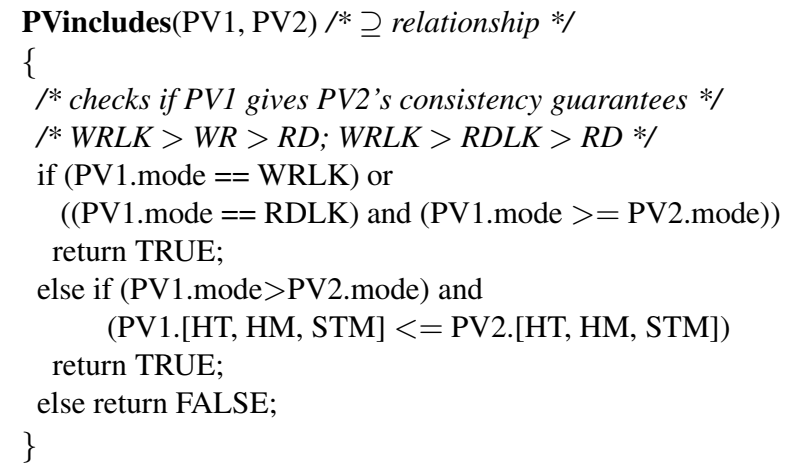

Figure 4: Comparing Privilege Vector guarantees.

CMs at neighboring replicas in the hierarchy exchange PVs based on client consistency demands, ensure that PVs do not violate guarantees of remote PVs, and push enough updates to preserve each other's PV guarantees. Although a Swarm server is responsible for detecting inaccessible peers and repairing replica hierarchies, its $\mathrm{CM}$ must continue to maintain consistency guarantees in spite of reorganization. To recover from unresponsive peers, the CMs at parent replicas grant PVs to children as leases, as explained below.

Privilege Vectors: A PV consists of four components that are independently enforced by different consistency mechanisms: an access mode (mode), a hard time/staleness limit (HT), a hard mod limit (HM) and a soft time+mod limit (STM.[t,m]). PVs are partially ordered by a PVincludes relation defined in Figure 4 that indicates how to compare the strength of guarantee provided by each of its components. By default, a file's root custodian starts with the highest PV ([WRLK, *, *, *] where $*$ is a wildcard), whereas a new replica starts with the lowest PV ([RD, $\infty, \infty, \infty])$. Associated with each replica of a file or file block is a current privilege vector (currentPV) that indicates the highest access mode and the tightest staleness and mod limit guarantees that can be given to local sessions without violating similar guarantees made at remote replicas.

A replica $\mathrm{R}$ remembers, for each neighbor $\mathrm{N}$, the relative PV granted to N (N.PVout) and obtained from N (N.PVin). The replica's currentPV is the lowest of the PVs it obtained from its neighbors. The relative PVs summarize to R, the consistency state of each neighbor's portion of the hierarchy. Since each replica only keeps track of the PVs of its neighbors, a replica's PV state is proportional to its fanout and not to the total number of replicas.

The bulk of the CM's functionality consists of the pull and push operations. If a client initiates a session that requires stronger consistency guarantees than the local replica has (e.g., the client requests the file in WRLK mode but the PV says it is read-only), the CM performs a pull operation. The pull operation obtains the consistency guarantees represented by a PV from those neighbors whose PVin is not strong enough to cover the PV, by 
issuing get messages to them in parallel. In response to a get message, those neighbors recursively pull the PV from their other neighbors, and recompute their own PV to be compatible with the new PV. Finally they reply with the new PV and any pending updates via put messages. By granting a PV, a replica promises to call back its neighbor before allowing accesses in its portion of the hierarchy that violate the granted PV's guarantee.

In contrast, a push operation is performed when there are updates available at a replica from either local clients or pushed from remote replicas that need to be passed along to other nodes in keeping with their consistency requirements. After the updates are applied locally, the local $\mathrm{CM}$ sends them via put messages to its neighbors if their divergence control requirements demand it.

To ensure fairness to client requests, concurrent pull operations for a particular file on a node are performed in FIFO order. As a replica issues get requests in parallel to neighbors in the hierarchy, pull latency grows logarithmically with the total number of replicas provided the hierarchy is kept dense by Swarm.

Enforcing Replica Divergence Bounds: To enforce a hard time bound (HT), a replica $\mathrm{R}$ issues a pull to neighbors with a write mode in their PV at most once every HT interval. A replica enforces a soft time bound (STM.t) by imposing a minimum push frequency (at least once per STM.t) on each of its neighbors. To enforce a modification bound of $\mathrm{M}$ unseen updates globally (HM and STM.m), a replica splits the bound into smaller bounds that are imposed on each of its neighbors that are potential writers. These neighbors may recursively split the bound to their children, which divides the responsibility of tracking updates across the replica hierarchy. A replica pushes updates whenever the number of queued updates reaches its local bound. If the mod bound is hard, the replica waits until the updates are applied and acknowledged by receivers before it allows subsequent updates.

Leases: To reclaim PVs from unresponsive neighbors, a replica always grants non-trivial PVs higher than [WR, $\infty, \infty, \infty]$ to its children as leases (time-limited privileges). The root node grants 60-second leases; other nodes grant slightly smaller leases than their parent lease to give them sufficient time to respond to lease revocation. A parent replica can unilaterally revoke PVs from its children (and break its callback promise) after their leases expire, which lets it recover from a lease-holding node becoming unavailable. Child replicas that accept updates using a leased privilege must propagate them to their parent within the lease period, or risk update conflicts and inconsistencies. Leases are periodically refreshed via a simple mechanism whereby a node pings other nodes that have issued it a lease for any data (four times per lease period in our current implementation). Each successful ping response implicitly refreshes all leases issued by the pinged node that are held by the pinging node. If a parent is unresponsive, the node informs its own children that they cannot renew their lease.

When a child replica loses contact with its parent while holding a lease, it reconnects to the replica hierarchy and issues a special 'lease recovery' pull operation. Unlike a normal pull, lease recovery prompts an ancestor of the unresponsive old parent to immediately renew the lease, without waiting for the inaccessible node's lease to expire. This "quick reconnect" is legal because the recovering node has holds a valid lease on the data and thus has the "right" to have its lease recognized by its new parent. This mechanism enables replicas to maintain consistency guarantees in the face of node failures and a dynamically changing replica hierarchy.

Update Propagation: Swarm propagates updates (modified file blocks or operational updates) via put messages. Each Swarm server stores operational updates in FIFO order in a persistent update log. For the purpose of propagation, each update from a client session is tagged by its origin replica and a node-local version number to identify it globally. To ensure reliable update delivery, a replica keeps client updates in its log in the tentative state and propagates them via its parent until a custodian acknowledges the update, switches the update to the saved state, handles further propagation. When the origin replica sees that the update is now saved, it removes the update from its local log.

To identify what updates to propagate and ensure exactly-once delivery, Swarm maintains a version vector $(V V)$ at each replica that indicates the latest update incorporated locally originating at every other replica. When two replicas synchronize, they exchange their VVs to identify missing updates. In general, the VV size is proportional to the total number of replicas, which could be very large (thousands), but since Swarm maintains replica trees and thus there is only one path between any two replicas in a stable tree topology, we can use compact neighbor-relative version numbers to weed out duplicate updates between already connected replicas. Servers exchange full version vectors only when a replica reconnects to a new parent, which occurs infrequently relative to update propagation events.

Ordering Concurrent Updates: When applying incoming remote updates, a replica checks if independent updates unknown to the sender were made elsewhere, indicating a potential conflict. Conflicts are possible when clients employ concurrent mode write (WR) sessions. Swarm forwards 'serially' ordered updates to the root custodian to be applied sequentially, avoiding conflicts. Swarm applies 'unordered' updates in their arrival order, which might vary from one replica to another, and ignores conflicts. For totally ordered updates, Swarm relies on a conflict resolution routine to impose a common global order at all replicas. This routine must apply the same update ordering criterion at all replicas to ensure a convergent final outcome. Swarm provides default reso- 
lution routines that reorder updates based on their origination timestamp (timestamp order) or by their arrival order at the root custodian (centralized commit order). The former approach requires Swarm servers to loosely synchronize their clocks via protocols such as NTP. The latter approach is similar to Bayou [3] and uses version vectors.

To enforce semantic ordering constraints (e.g., atomic or causal), Swarm tags each update with the ordering constraint of the issuing session. A CM uses its local update log to determine in what order it must propagate or apply updates to satisfy these ordering constraints. Atomically grouped updates are always propagated and applied together.

\section{Evaluation}

In Section 6.1, we show how Swarm's CC implementation of close-to-open semantics exploits 'nearby replicas' (similar to Pangaea [14]) to outperform Coda's client-server implementation in a sequential file sharing (roaming) scenario. In Section 6.2 we demonstrate how the performance and scalability of a replicated BerkeleyDB database varies under five consistency mechanisms that range from 'strong' (appropriate for a conventional database) to 'time-based' (appropriate for typical directory services). Swarm offloaded the hard problems of replication and consistency management from both applications, thereby simplifying their implementation while providing them with a rich set of consistency choices.

For all experiments, we used the Emulab Network Testbed [17] to emulate WAN topologies among clustered PCs. The PCs have 850Mhz Pentium-III CPUs with 512MB of RAM and run Redhat Linux 7.2. Swarm servers run on each PC as user-level processes and store files in a single directory in the local file system, using the SWID as the filename. The Swarm replica fanout was configured to a low value of 4 to induce deep multilevel replica hierarchies.

\subsection{Swarmfs: A Flexible Distributed File System}

We evaluate a Swarm-based file system (Swarmfs) on a synthetic roaming benchmark across an emulated non-uniform WAN network shown in Figure 5. In this benchmark, we model collaborators at a series of locations accessing shared files, one location at a time. This type of 'sequential file sharing' is representative of mobile file access or work-flow applications where collaborators take turns updating shared documents or files. We compare Swarmfs to Coda employing both weak (coda-w) and strong connectivity modes (coda-s). Like Pangaea, and unlike Coda, Swarm incurs low latency by treating replicas as peers for consistency enforcement. Unlike Pangaea, Swarmfs guarantees closeto-open consistency. To support close collaboration or near-simultaneous file access, users require close-

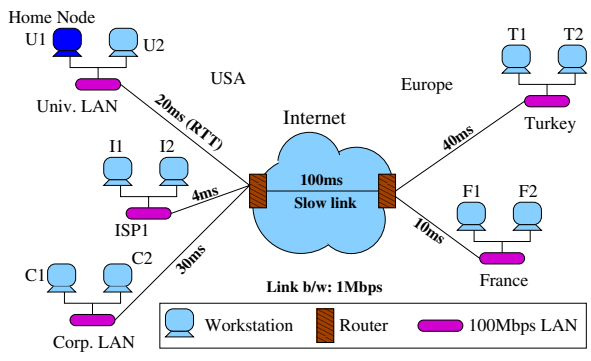

Figure 5: Network topology for Swarmfs roaming experiment.

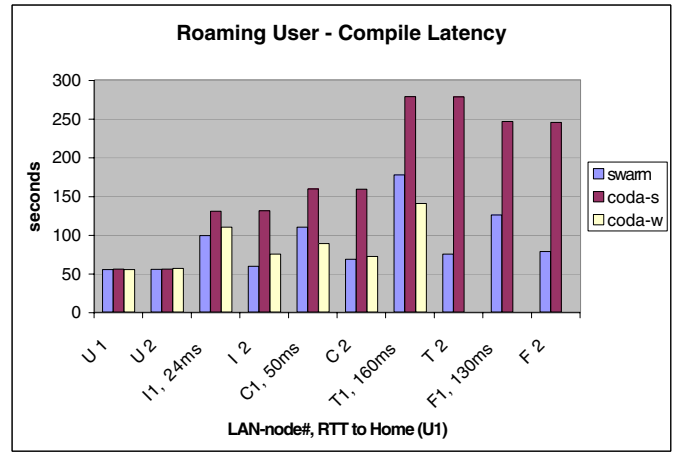

Figure 6: Roaming File Access: Swarmfs pulls source files from nearby replicas. Strong-mode Coda correctly compiles all files, but exhibits poor performance. Weak-mode Coda performs well, but generates incorrect results on the three nodes farthest from the server (U1).

to-open consistency semantics even when weakly connected.

The topology modeled consists of five widely distributed campuses, each with two machines on a $100 \mathrm{Mbps}$ LAN. The node U1 (marked 'home node') initially stores the Tcl- 8.4 source files: 163 files with a total size of 6.7MB. We run Swarmfs servers and Coda clients on all nodes. The Coda server runs on U1.

In our synthetic benchmark, clients at various nodes sequentially access files. Each client modifies a source file, compiles the Tcl- 8.4 source tree, and then deletes all object files. These operations represent isolated updates, intensive file-based computation, and creating and deleting many temporary files. Clients on each campus node (in the order University (U) $\rightarrow$ ISP1 (I) $\rightarrow$ Corporate $(\mathrm{C}) \rightarrow$ Turkey $(\mathrm{T}) \rightarrow$ France $(\mathrm{F}))$, perform the edit-compile-cleanup operation one after another.

Figure 6 shows the compilation times on each node. Since Swarm creates efficient replica hierarchies and acquires files from nearby replicas (e.g., from another node on the same LAN, or, in the case of France, from Turkey), it outperforms Coda's client-server implementation, which always pulls files from server U1.

In weak connectivity mode (coda-w), Coda guarantees only eventual consistency, which causes incorrect 


\begin{tabular}{c|c}
\hline Consistency Semantics & CC options \\
\hline locking writes & WRLK \\
master-slave writes & WR, serial \\
close-to-open rd, wr & RD/WR, time=0, hard \\
time-bounded rd, wr & time $=10$, hard \\
optimistic/eventual rd, wr & RD/WR, time $=0$, soft \\
\hline
\end{tabular}

Table 2: Consistency flavors employed for Replicated BerkeleyDB and the CC options to achieve them. The unspecified options are set to $[\mathrm{RD} / \mathrm{WR}$, time $=0, \bmod =\infty$, soft, no semantic deps, total order, pessimistic, session visibility \& isolation].

behavior starting at node T2. In this case, coda-w's trickle reintegration causes the client on $\mathrm{T} 1$ to eagerly push huge object files to U1, thereby clogging its network link and delaying notification of subsequent file deletions. By the time T2 sees T1's file deletion operations, it has already started its compile and used stale object files. Coda's strong connectivity mode provides close-to-open semantics but incurs double the latency of Swarmfs because of write-through to the server (U1). Both these problems occur because Coda servers never pull updates from clients, but rather clients must push updates to the server. Swarm avoids this by employing the same pull mechanism uniformly at all replicas.

\subsection{SwarmDB: Replicated BerkeleyDB}

Popular databases (e.g., mySQL, Oracle, and BerkeleyDB) predominantly employ master-slave replication (if any) across the wide area due to its simplicity; readonly replicas are deployed near clients to scale query performance, but updates are applied at a central master site to ensure serializability. For applications that can handle concurrent updates (e.g., many directory services), master-slave replication is overly restrictive and does not scale or exploit regional locality. By using Swarm to implement database replication, we can choose on a per-client basis how much consistency is required. High throughput can be achieved when the consistency requirements are weaker, as in a directory service [9], while the same code base can be used to provide a strongly consistent database when required.

We augmented the BerkeleyDB embedded database library [15] with replication support as a wrapper library called SwarmDB. A typical SwarmDB-based application is shown in Figure 1. SwarmDB stores a BerkeleyDB database in its native format in a Swarm file and intercepts BerkeleyDB update operations to invoke them as operational updates on the local Swarm server via a SwarmDB plugin. SwarmDB-based application instances access the database via Swarm servers spread across a WAN that cache the database locally.

We measure SwarmDB's read and write throughput under a full-speed update-intensive workload when employing the five distinct flavors of consistency semantics shown in Table 2. We compare the performance of the five flavors of SwarmDB against BerkeleyDB's

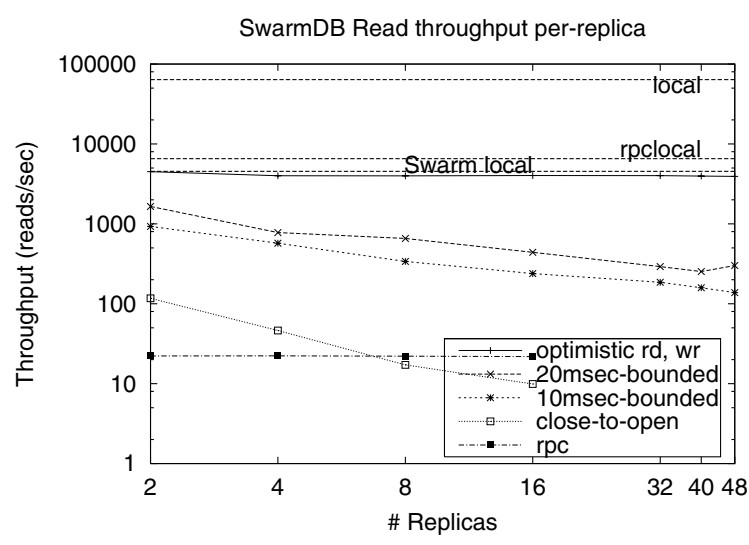

Figure 7: SwarmDB Per-replica Read Throughput

client-server (RPC) implementation. The consistency flavors (listed from strongest to weakest) are: (1) locking writes and optimistic reads, where writes are serialized via locks before being propagated, (2) master-slave writes and optimistic reads, where all writes are serially ordered at the root of the replica hierarchy before propagation, (3) close-to-open consistency, (4) time-bounded staleness, where data is synched before access if more than a threshold time has passed since the last synch, and (5) optimistic writes and reads, where writes are performed locally before being propagated to other replicas. Our synthetic benchmark creates and populates a BerkeleyDB database with 1000 key-value pairs inside a Swarm file. The database size does not affect replication performance except during startup since we employ operational updates, where Swarm replicates the entire database file as a single consistency unit and propagates operations instead of changed data.

We run the benchmark on 2 to 48 nodes. Nodes are each connected by a $1 \mathrm{Mbps}, 10$-msec delay WAN link to a backbone router, which implies a $40 \mathrm{~ms}$ roundtrip between any two nodes. Each server executes 10,000 random operations at full-speed, i.e., with no think time. The operation mix consists of 5\% adds, $5 \%$ deletes, $20 \%$ updates, 30\% lookups, and 40\% cursor-based scans. Reads (lookups and cursor-based scans) are performed directly on the database file, while writes (adds, deletes, and updates) are sent to the local Swarm server by the SwarmDB library. Each operation opens a Swarm session on the database file in the appropriate mode, performs the operation, and closes the session.

Figures 7 and 8 show the average throughput observed per replica for reads and writes. In addition to the SwarmDB results, we present baseline performance when directly operating on a database file stored in the local file system (local), when invoking RPCs to a colocated BerkeleyDB server (rpclocal), and when accessing a local Swarm-based database file with no replication (Swarm local). Swarm local represents the best throughput possible using SwarmDB with our Swarm prototype. 


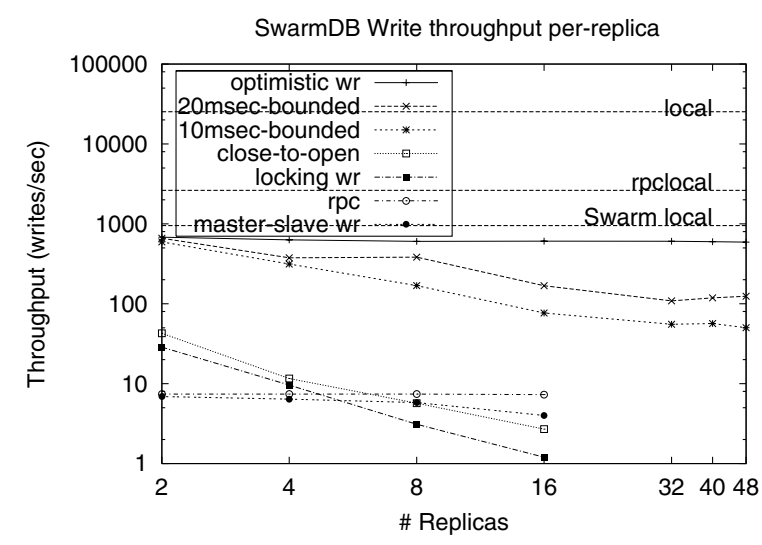

Figure 8: SwarmDB Per-replica Write Throughput

The high cost of IPC between the client and server cause rpclocal and Swarm local to perform poorly compared to local.

Figure 7 shows that read throughput scales well when we request soft (push-based) or time-based consistency guarantees, but not when we request hard (firm pullbased) guarantees, as expected. Due to the updateintensive nature of the workload, there is almost always a write in progress somewhere in the system. Thus the strict pull-based schemes are constantly pulling updates across WAN links, suffering the same high latency that the RPC-based solution incurs. As a result, close-toopen and strong consistency do not scale beyond 16 replicas, given the high degree of write-sharing. Eventual consistency scales well to large replica sets even when pushing updates eagerly, because Swarm enables the DB plugin to remove self-canceling updates. Tolerating even a small amount of staleness (10ms) significantly improves read and write performance over closeto-open consistency, because the cost of synchronization over the wide area is very high, and amortizing it over multiple operations has substantial latency benefit.

In summary, different consistency options provide vastly different semantics and performance characteristics for the same workload. Composable consistency enables an application to choose the right semantics based on its specific need at hand.

\section{Conclusions}

In this paper we proposed a new way to structure consistency management for $\mathrm{P} 2 \mathrm{P}$ sharing of mutable data, called composable consistency. It splits consistency management into design choices along several orthogonal dimensions and lets applications express their consistency requirements as a vector of these choices on a per-access basis. The design choices are orthogonal and can be combined in various ways to yield a rich collection of semantics, while enabling efficient implementation. We outlined an implementation of the model in a pervasive peer replication environment spanning non- uniform networks. Our evaluation showed how composable consistency is both expressive and practical.

\section{Acknowledgments}

We thank the anonymous reviewers for their valuable comments on an earlier draft of this paper. We are especially grateful to Jay Lepreau for his support to the Swarm project, to Mike Hibler and Eric Eide for helping improve this paper with thorough feedback, and to Rob Ricci and Kirk Webb for providing excellent support for our Emulab-based experiments.

\section{References}

[1] M. Blaze. Caching in Large Scale Distributed File Systems. PhD thesis, Princeton University, 1993.

[2] L. Cox and B. Noble. Fast reconciliations in Fluid Replication. In Proc. 21 st Intl. Conference on Distributed Conputing Systems, Apr. 2001.

[3] A. Demers, K. Petersen, M. Spreitzer, D. Terry, M. Theimer, and B. Welch. The Bayou architecture: Support for data sharing among mobile users. In Proceedings of the Workshop on Mobile Computing Systems and Applications, Dec. 1994.

[4] J. Howard, M. Kazar, S. Menees, D. Nichols, M. Satyanarayanan, R. Sidebotham, and M. West. Scale and performance in a distributed file system. ACM Transactions on Computer Systems, 6(1):51-82, Feb. 1988.

[5] J. Kistler and M. Satyanarayanan. Disconnected operation in the Coda file system. In Proc. 13th Symposium on Operating Systems Principles, pages 213-225, Oct. 1991.

[6] N. Krishnakumar and A. Bernstein. Bounded ignorance: A technique for increasing concurrency in a replicated system. ACM Transactions on Data Base Systems, 19(4), Dec. 1994.

[7] R. Ladin, B. Liskov, L. Shrira, and S. Ghemawat. Providing high availability using lazy replication. ACM Transactions on Computer Systems, 10(4), 1992.

[8] C. Lamb, G. Landis, J. Orenstein, and D. Weinreb. The Objectstore database system. Communications of the ACM, Oct. 1991.

[9] Microsoft Corp. Active directory (in windows 2000 server resource kit). Microsoft Press, 2000.

[10] A. Muthitacharoen, B. Chen, and D. Mazieres. Ivy: A read/write peer-topeer file system. In Proc. 5th Symposium on Operating System Design and Implementation, Dec. 2002.

[11] M. Nelson, B. Welch, and J. Ousterhout. Caching in the Sprite network file system. ACM Transactions on Computer Systems, 6(1):134-154, 1988.

[12] P. Reiher, J. Heidemann, D. Ratner, G. Skinner, and G. Popek. Resolving file conflicts in the Ficus file system. In Proceedings of the Summer Usenix Conference, 1994.

[13] A. Rowstron and P. Druschel. Storage management and caching in PAST, a large-scale, persistent peer-to-peer storage utility. In Proc. 18th Symposium on Operating Systems Principles, 2001.

[14] Y. Saito, C. Karamanolis, M. Karlsson, and M. Mahalingam. Taming aggressive replication in the Pangaea wide-area file system. In Proc. 5th Symposium on Operating System Design and Implementation, pages 1530, 2002.

[15] Sleepycat Software. The BerkeleyDB database. http: //sleepycat. $\mathrm{com} /, 2000$.

[16] S. Susarla and J. Carter. Middleware support for locality-aware wide area replication. Technical Report UUCS-04-017, University of Utah School of Computer Science, Oct. 2004

[17] B. White, J. Lepreau, L. Stoller, R. Ricci, S. Guruprasad, M. Newbold, M. Hibler, C. Barb, and A. Joglekar. An integrated experimental environment for distributed systems and networks. In Proc. 5th Symposium on Operating System Design and Implementation, Boston, MA, Dec. 2002.

[18] H. Yu and A. Vahdat. Design and evaluation of a continuous consistency model for replicated services. In Proc. 4th Symposium on Operating System Design and Implementation, Oct. 2000. 